\title{
Innover en aménagement du territoire: propositions et questions à l'intention des aménagistes
}

\author{
Une contribution au débat sur l'aménagement
}

This article is a contribution to the discussion of spatial planning in Switzerland. The seventies and nineties were significant years regarding the goals of spatial planning: the realisation of policies and legislation. Working out the necessary tools for planning and their realisation during the boom years with a clear and active role of the State.

For the last 10 years spatial planning has been experiencing some difficulties in its double-role of policy making: to coordinate and setting goals. The legal complexities, the influence of the economy and the problematic role of the State explain partly the situation. However, these difficulties are also the responsibilities of the experts in spatial planning.

The following four areas are dealt with:

- The necessity to take the new values set by society into consideration as well as the high standards required, forcing spatial planning to take action accordingly.

- The new kind of collaboration between the economy and spatial planning, and for the latter to become a profitable and safe asset for the economy.

- The necessity for spatial planning to build alliances with various political bodies thus promoting cooperation between them.

- An examination of the nature of spatial planning's performance and its target group.

La rédaction de la revue DISP souhaite alimenter le débat d'idées autour du rôle de l'aménagement dans notre pays. C'est le but des réflexions proposées. Dans cet article, nous concevons l'aménagement du territoire comme une politique publique qui exerce une double mission (cf. à ce sujet l'étude 1998 de R. Jagmetti pour le compte de I'O FAT):
- assurer la coordination entre les niveaux institutionnels en charge de l'aménagement (communes, cantons et Confédération) et entre les politiques publiques ayant un impact sur le territoire (notamment l'agriculture, les transports, la politique régionale, la protection de l'environnement); c'est la «Q uerschnittsfunktion» comme on le dit en Suisse alémanique;

- régler l'affectation du sol à travers les plans de zones et les règlements, notamment de construction; c'est la dimension sectorielle de l'aména gement.

Pendant les années 70-90, l'aménagement a, sans a ucun doute, connu une période faste. Mais depuis quelques années, l'heure est au doute quant à son rôle et sa finalité. L'aménagement est en perte de vitesse, il ne serait plus en adéquation avec le temps, il peine à faire reconnaître ses prestations et les aménagistes s'interrogent sur leur rôle et sur leur pratique. Fin d'une période heureuse dans l'attente de jours meilleurs? Inadéquation d'instruments aux besoins et problèmes nouveaux de notre société? $\mathrm{O} u$ alors crise d'une politique publique et d'une pratique professionnelle, toutes deux en difficulté d'adaptation dans une nouvelle réalité politique, sociale et économique?

Nous n'avons ni la prétention d'exhaustivité ni celle d'apporter des réponses toutes faites. Le propos n'est pas de «faire la leçon», mais bien plus de questionner pour susciter la discussion parmi les spécialistes et responsables de l'a ménag ement [1].

1970 - 1990: les années «glorieuses» de l'aménagement du territoire

Indiscutablement, ce sont des années fastes:

- la législation se met en place, certes non sans difficultés, tant a u niveau cantonal que fédéral; la loi fédérale entre en vigueur le 1 er janvier 1980; l'aménagement du territoire bénéficie d'une légitimité et d'appuis politiques; les débats sont vifs; il n'y a pas unanimité, mais les divergences portent essentiellement sur les modalités d'application de la législation, sur son champ d'action, sur la répartition des compétences entre Confédération et cantons et non sur ses finalités et sa nécessité;

- à travers la planification, l'Etat s'exerce à la coordination de ses politiques sectorielles; il place beaucoup d'espoirs dans les plans directeurs, les plans sectoriels pour harmoniser ses activités; c'est l'époque des conceptions directrices (transports, énergie, etc.) et des concepts d'aménagement et de développement;

- la planification a la cote; les experts, donc les aménagistes, disposent d'une crédibilité, tant auprès des responsables politiques que des citoyens; et l'Etat est, à cette époque, un très grand "consommateur» d'exercices de planification;

- et surtout, les plans d'affectation a vec leurs règlements d'application sont adoptés par la quasi totalité des communes suisses.

Le bilan est globalement positif, même s'il y aurait des nuances à apporter. C'est ainsi, par exemple, que l'élaboration des plans directeurs cantonaux et surtout leur mise en œuvre, se révélera un exercice plus laborieux que prévu; pour l'affectation, on constate le surdimensionnement de la zone à bâtir dans de nombreuses communes; la gestion de la zone agricole ne se fait pas sans heurts. Mais en vingt ans, l'aménagement a gagné ses lettres de noblesse parmi les politiques publiques.

1990-1998: les années de remise en cause et de doute

Au cours de ces dernières années, l'aménagement se voit contester ses finalités et ses prérogatives; il rencontre des difficultés pour exercer sa double mission de politique publique: la coordination et l'affectation.

L'aménagement peine à assurer ses tâches de coordination: les mutations institutionnelles en cours (remise en cause du fonctionnement du fédéralisme, regroupements des communes, émergence de régions à géométrie variable) introduisent beaucoup d'incertitudes (on ne sait plus qui fait quoi); plusieurs politiques publiques (agriculture, santé, 
transports, etc.) se restructurent actuellement sous la contrainte budgétaire et en poursuivant des logiques sectorielles. Le territoire est le grand oublié de la restructuration et avec lui l'aménagement.

La mise en œuvre des plans communaux adoptés se heurte à des difficultés: les affectations planifiées par l'autorité ne correspondent plus aux utilisations souhaitées par les propriétaires. Les exemples abondent pour illustrer ce constat: le type de zone à bâtir est contesté (on a planifié un habitat groupé et les propriétaires souhaitent réaliser des villas, des plans de quartier ne se concrétisent pas); les solutions préconisées pour les surfaces commerciales sont contestées; et le débat autour des constructions admises en zone agricole s'inscrit dans ce contexte de critique de l'aménagement.

A ujourd'hui, l'aménagement souffre d'un déficit de légitimité politique et sociale. Les priorités vont à l'emploi, au social, à l'équilibre budgétaire. II est devenu une politique publique «secondaire» pour les responsables politiques fédéraux et cantonaux et il n'assume que très difficilement sa fonction de coordination. Cette dernière a été accaparée par la politique financière: to ut se mesure à l'aune des budgets et des comptes. Là également, il y a des nuances à apporter. L'aménagement est partie prenante dans la coordination de projets concrets (pôles de développement économique, projets routiers, gravières, etc.); le plan directeur cantonal est un outil utile pour la négociation avec la Confédération et ses politiques sectorielles. Mais dans le débat politique actuel concernant la réorientation des politiques publiques (répartition des tâches, des compétences entre Confédération, cantons et communes; péréquation financière), l'aménagement est très discret.

\section{Pourquoi cette perte de crédibilité de l'a ménagement?}

Il y a un discours usuel dans le monde des aménagistes qui tient en quelques mots: le politique a démissionné, l'économie dicte sa loi, et les juristes déci- dent. Affirmations à la hache, mais qui résument bien le débat d'idées autour de la crise actuelle de l'aménagement du territoire en Suisse.

Les autorités sont avant tout préoccupées par l'équilibre budgétaire et les contraintes financières. II n'y a plus de coordination des politiques sectorielles, si ce n'est sous l'angle financier. Les responsables politiques cantonaux se réfèrent au plan directeur quand il sert leurs intérêts; on n'hésite pas à faire preuve de souplesse dans les autorisations et dérogations par opportunisme politique.

Les autorités communales ont adopté un plan d'aménagement, mais elles ont tendance à l'oublier dans les mois qui suivent. Elles invoquent des arguments économiques pour justifier l'implantation d'une usine ou d'un centre commercial en contradiction avec les intentions de leur plan d'affectation. Les critères d'aménagement ne font pas le poids face aux propositions de création d'emplois et d'investissements. Quant au citoyen, il est plus soucieux de son intérêt personnel à court terme que de l'intérêt général à long terme.

Les juristes sont devenus la référence incontournable puisque constamment sollicités à la suite de recours et d'oppositions. A cela s'ajoute la complexité liée à la multiplicité des législations concernant le territoire qui fait que l'aménagement est souvent perçu - à tort ou à raison - comme une affaire de juristes.

Cette analyse contient sa part de vérité. $M$ ais elle fait la part belle aux spécialistes de l'aménagement, car elle donne à penser que l'essentiel des difficultés rencontrées actuellement en aménagement trouve son origine à l'extérieur, chez les autres (le politique, le juriste, l'économiste, le chef d'entreprise, le citoyen). N 'y aurait-il pas d'autres causes dont la responsabilité appartient aux spécialistes? Je propose quatre domaines ou pistes de réflexion pour alimenter le débat:

- à temps nouveaux, nouvelles conceptions et nouvelles démarches d'aménagement?

- liens entre aménagement et économie: des frères ennemis aux sœurs siamoises?
- politique d'aménagement du territoire: à la recherche de nouvelles alliances politiques;

- et si l'aménagement raisonnait et travaillait en termes de prestations?

Ce sont des domaines où les spécialistes de l'a ménagement peuvent et doivent faire preuve d'imagination et de créativité. Les réflexions s'adressent à l'ensemble des aménagistes, même si celles développées à propos des nouvelles alliances politiques et des prestations concernent plutôt ceux d'entre eux qui travaillent dans le secteur public.

\section{Les temps ont changé et l'aména- gement n'en tire pas toutes les conséquences}

L'aménagement du territoire s'est développé dans une période (les années 1970-90) dominée par la rationalité technique et économique. C'est une période de certitudes pour l'aménagement et sa finalité est évidente pour tous: il doit viser l'affectation efficace et cohérente des ressources disponibles et l'optimisation du fonctionnement des structures sociales. Cette finalité relève du fonctionnalisme. Les théories et les pratiques s'appuient sur la méthode scientifique (positivisme) qui présente les apparences de l'objectivité et de la rationalité. L'aménagement du territoire s'apparente à une ingénierie technique et fonctionnelle où il suffit de suivre un certain nombre de prescriptions pour réaliser un projet social cohérent, objet d'un large consensus auprès de l'ensemble de la population. Cette conception de l'aménagement suppose soit un pouvoir centralisé et dépositaire de l'intérêt général (cas de la France), soit une forte adhésion autour d'un projet s'inscrivant dans une logique de progrès social et économique (cas de la Suisse). Beaucoup d'aménagistes sont encore imprégnés de cette philosophie et comptent sur des temps futurs qui leur seront plus favorables.

$0 r$, a u cours de ces dernières années, les temps ont changé rapidement et de manière profonde. Un monde nouveau se construit, aux frontières incertaines, caractérisé par des conflits de valeurs et d'intérêts, par une mobilité extraor- 
dinaire des personnes, des biens, des services et surtout de l'information. Le marché du travail et les modes de fonctionnement des entreprises changent radicalement. Le rôle de l'Etat est mis en discussion. De nouvelles règles émergent en matière de répartition des compétences et des ressources entre le secteur public et le secteur privé; le jeu d'acteurs est de plus en plus complexe et se joue sur plusieurs scènes publiques et privées. La distinction entre secteur public et secteur privé devient floue. La concurrence se développe non seulement entre les entreprises, mais également entre les régions, les villes. Ces mutations sont à l'origine de nouvelles modalités d'appropriation et d'occupation du sol. L'aménagement, et surtout l'aménagiste, n'ignore pas ces changements. $M$ ais en a-t-il tiré toutes les conséquences pour la pratique? Je n'en suis pas si sûr.

L'ambition de l'aménagement est de traduire dans l'espace les visions, les objectifs et les besoins de la société. Pour réaliser cette ambition, l'aménagement utilise des instruments comme la conception directrice, le concept d'urbanisation et de développement, le plan directeur, le plan d'affectation; il propose des règles du jeu à travers des procédures de planification et de décision. Mais cette ambition n'est légitime que s'il est à même de prendre en compte et d'aider à la réalisation des projets des acteurs tant privés que publics, sinon ses projets et plans resteront du domaine des intentions.

Si l'aménagement du territoire veut avoir, à l'a venir voix au chapitre, il doit remettre en cause son approche fonctionnaliste. Il doit être en mesure de prendre en compte les nouveaux besoins de notre société et de proposer de nouvelles démarches de planification. Sinon, la société organisera son territoire en confinant l'aménagement à de simples tâches réglementaires liées au zonage et au règlement de construction.

\section{Prendre en compte les nouvelles valeurs} et les besoins qualitatifs de la société

Un peu schématiquement, on peut dire que l'aména gement a tenté de rationaliser les besoins des acteurs en surfaces, en volumes, en distances, les traduisant en zones et en prescriptions réglementaires. II ne s'agit pas de contester cette dimension de l'aména gement. $\mathrm{M}$ a is depuis les années 80 , ces besoins n'ont plus seulement cette dimension fonctionnelle et rationnelle; ils font référence à de nouvelles valeurs où émergent des préoccupations de qualité de vie, d'identité individuelle et sociale, d'éthique et de bonheur. Ces préoccupations, de nature qualitative et existentielle, sont en train de modifier (surtout à partir des années 90) le comportement de la population dans son appropriation de l'espace. L'aménagement s'inscrit dans une société qui hésite entre l'ordre et la liberté, entre l'individuel et le collectif; une société de plus en plus urbaine et non plus rurale (on ne doit pas oublier qu'en Suisse, l'aménagement privilégie largement les intérêts de la seconde).

O r l'aménagement du territoire peine à prendre en compte ces nouveaux besoins et comportements dans sa pratique - au-delà des grandes déclarations d'intention -, faute d'instruments adéquats à cet effet. Un projet d'aménagement peut être rationnel, fonctionnel. Peut-on envisager un aménagement relationnel, émotionnel, sensuel? Les plans directeurs, les plans sectoriels, les plans d'affectation conserveront leur utilité à l'a venir. M ais comment les concevoir et surtout les mettre en œuvre pour tenir compte des valeurs et surtout des nouveaux comportements - notamment en matière de mobilité - qui se dessinent dans la société? C'est le défique doit relever l'aménagement de demain.

\section{Promouvoir un aménagement processuel}

Une partie de la réponse se trouve dans la manière de concevoir la démarche d'aménagement. Ce dernier n'est pas un but en soi, il est un moyen au service des intérêts et des valeurs de ses acteurs publics et privés de la société. L'aménagement du territo ire doit avoir pour finalité la médiation des conflits liés à l'utilisation du sol. II doit alors prendre en compte la multiplicité de ces valeurs et composer avec un intérêt public compo- site et fluctuant. II est à concevoir comme une démarche visant à intégrer les conflits inhérents à notre société et à les arbitrer à partir de la pesée des intérêts en présence. II a ppartient à l'a ménagiste de faire ressortir les enjeux et les termes de la négociation à mener pour faire aboutir le projet public d'aménagement intégrant les projets particuliers. Cette approche n'est pas nouvelle, cependant la réflexion mériterait d'être poussée un peu plus loin.

Un constat s'impose. Malgré des efforts évidents en matière de participation et d'information du citoyen, la démarche d'a ménagement est encore largement perçue comme un acte technique et juridique. II y a une procédure à suivre et des compétences à respecter. Cette perception normative est souvent partagée au sein de la population. Peu importe qu'elle soit simpliste, elle contribue largement à la «mauvaise réputation» de l'aménagement. La pratique participative doit s'intensifier, mais en levant des ambiguités quant aux objectifs de la participation.

En effet, la participation est encore souvent conçue comme un moyen pour convaincre la population, pour vendre le «bon» projet, ou pour faire passer un certain nombre de valeurs cachée dans un projet d'aménagement. L'aménagiste n'est pas neutre. II véhicule des valeurs qui se retrouvent dans ses propositions. Sous les étiquettes de qualité de la vie, de protection de l'environnement, de développement durable, se cachent des valeurs et une idéologie. II ne s'agit pas de contester à l'aménagiste le droit de défendre ses valeurs ni de le traiter de manipulateur à travers son a ctivité. II lui appartient par contre de s'interroger sur son rôle dans la conception et la démarche d'aménagement du territoire.

Les débats que suscite l'implantation de grands centres commerciaux permettent d'illustrer ce questionnement. S'agitil pour l'aménagiste 1) de contester le principe des grands centres commerciaux 2), de s'y opposer au profit d'un autre type de commerces 3 ), de veiller à leur localisation optimale 4), de s'assurer que leur implantation respecte la législation 5), de mettre sur la table l'ensemble des incidences d'une telle 
implantation? La pratique révèle des ambiguïtés parmi les professionnels de l'aménagement. La démarche cache souvent leur souci de faire passer leurs valeurs, c'est-à-dire leur appréciation des centres commerciaux. Ceci explique en partie la méfiance manifestée à l'égard de l'aménagement par les milieux de la construction, des transports privés et de l'économie en général.

Aire de gestion et de médiation des conflits, cette conception privilégie une approche processuelle de l'aménagement soucieuse de l'efficacité politique et sociale. Elle implique une très grande rigueur et une transparence absolue des procédures et des processus de décision. Elle nécessite une approche en terme de variantes a vec leurs avantages et leurs inconvénients pour chacun des acteurs concernés. L'approche est exigeante pour les professionnels; ces derniers sont encore souvent préoccupés par «la bonne» solution - c'est-à-dire celle qu'ils privilégient -; ils réduisent l'aménagement processuel à une simple démarche d'information du citoyen. $0 \mathrm{r}$ celui-ci veut avoir prise sur le processus de décision (d'où ses exigences de consultation/concertation). A l'avenir, le succès de l'aménagement sera surtout fonction de la qualité d'adhésion à ses propositions (elles sont à même de répondre aux besoins des acteurs concernées) alors que les aménagistes privilégient encore trop la qualité intrinsèque des projets comme élément clé $d u$ succès.

\section{A ménagement et économie: des frères ennemis aux sœurs siamoises}

\section{Des relations par nature conflictuelles}

Les relations entre a ména gement et économie ont été et seront toujours conflictuelles. L'économie est faite de décisions très diversifiées prises par de nombreux acteurs indépendants les uns des autres, en situation de concurrence et soumis à des préoccupations de rentabilité à court terme. L'aménagement du territoire doit prendre en compte l'ensemble des intérêts et des valeurs qui sont associés à l'utilisation du sol. O n constate ainsi une tension constante entre les préoccupations de l'aménagement soucieux de définir des règles valables pour le plus grand nombre dans une perspective à long terme - et les exigences de l'économie - guidées par des considérations individuelles à court terme.

La nature conflictuelle de ces relations est favorisée par une méconnaissance et une attitude de méfiance réciproques. Les milieux économiques tendent à se faire une opinion sur l'aménagement à partir de quelques cas litigieux et procèdent par généralisations souvent abusives. Les procédures et les instruments d'a ména gement sont considérés comme visant uniquement à interdire, et leur apport en terme de gains ou de facilités pour le secteur privé est généralement ignoré. Les milieux de l'aménagement, pour leur part, méconnaissent largement les règles de fonctionnement de l'économie, souvent amalgamées aux règles du promoteur immobilier. II en résulte un discours peu cohérent, où les relations avec l'économie se limitent à des énumérations de problèmes hétéroclites, à partir desquels le développement d'une stratégie apparaît impossible. On notera que de nombreux services cantonaux d'aménagement ont publié des guides et manuels sur la manière de prendre en compte l'écologie dans l'aménagement; mais on cherche en vain un manuel sur la manière de considérer les aspects économiques et de finances publiques.

Cette situation est le résultat de la pratique de l'aménagement dans les années de croissance économique: les préoccupations étant alors surtout de canaliser les investissements privés et publics, la recherche d'un partenariat explicite, avec les milieux économiques n'apparaissait pas nécessaire. Aujourd'hui, cependant, dans le contexte de restructuration que nous connaissons, des relations étroites et nouvelles doivent être établies et entretenues a vec les milieux économique si l'on souhaite assurer à notre pays un développement durable et une organisation territoriale de qualité, tout en intégrant les nouveaux besoins des acteurs économiques.

\section{Un aménagement qui doit mettre l'investissement privé au cœur de ses préoccupations}

La législation définit les relations entre l'a ména gement et l'économie. C'est ainsi que l'art. 1, al. 2 de la LAT demande aux collectivités:

- de créer et de maintenir un milieu bâti harmonieusement aménagé et favorable à l'habitat et à l'exercice des activités économiques;

- de favoriser la vie sociale, économique et culturelle des diverses régions du pays et de promouvoir une décentralisation judicieuse de l'urbanisation et de l'économie.

Pour atteindre ces objectifs, l'aménagement s'appuie sur ses instruments traditionnels de type réglementaire et de planification ainsi que sur l'ensemble des procédures de décision permettant la gestion du territoire. II tente aussi de développer des prestations de conseils, préconise de nouvelles zones d'activité, fonde des espoirs sur des instruments conformes à l'économie de marché. $M$ a is les aménagistes ont tendance à oublier que ces instruments sont impuissants, à eux seuls, à assurer la concrétisation des objectifs de la planification. Ces objectifs ne seront atteints que si l'investissement privé vient concrétiser les projets et propositions d'aménagement.

Un plan approuvé ne signifie pas qu'il sera appliqué, et il ne suffit pas qu'un canton légalise une zone industrielle ou un pôle d'activités pour qu'ils se remplissent d'entreprises pourvoyeuses d'emplois. II faut que des agents économiques (entrepreneurs, banquiers, promoteurs, investisseurs, etc.) décident d'investir dans des activités et projets industriels, tertiaires, touristiques ou immobiliers selon la planification souhaitée. $\mathrm{Or}$ leurs décisions sont fonction essentiellement des perspectives de rentabilité de leurs investissements, perspectives étroitement conditionnées par l'environnement national et international défini par les règles du marché (en termes de contraintes imposées et d'opportunités offertes). L'aménagement est tributaire des décisions de l'investisseur privé. 
Cet état de fait pouvait être éludé en période de haute conjoncture: jusque dans les années 80, l'aménagement a été mis en œuvre dans un contexte de croissance économique. Celle-ci s'est traduite, pour les entreprises, par des possibilités d'investissements importants ayant de bonnes perspectives de rentabilité et, pour les cantons, par les résultats satisfaisants de leur politique économique. Pour l'a ména gement, il s'agissait a vant to ut de canaliser spatialement la croissance et d'en limiter les conséquences négatives. Des instruments ont été développés à cet effet, qu'il s'agisse de plans ou de dispositions réglementaires. Les investisseurs privés se présentant nombreux pour s'implanter dans les zones qui leur étaient affectées, le territoire a largement pu être aménagé conformément à la planification (il y aurait des nuances à apporter par domaine ou région). La bonne santé financière des collectivités publiques a en outre permis la concrétisation de mesures d'aménagement favorables à la protection du paysage et de l'environnement.

A ujourd'hui, cependant, le cadre économique se caractérise par une rupture importante. La globalisation implique une concurrence accrue entre les entreprises, et cela même dans des secteurs protégés. Les entreprises réalisent leur mutation, à travers fermetures, délocalisations et restructurations. Leur échelle spatiale de référence se modifie (marchés, fournisseurs, recrutement du personnel, partenaires de sous-traitance, accès à la technologie, etc.), de même que leurs conditions d'implantation dans le territoire. La capacité à fonctionner en réseau et à accéder aux informations pertinentes prime sur les facteurs traditionnels de localisation, généralement privilégiés par les stratégies d'a ména gement.

\section{Un aménagement facteur de gains et de sécurité pour l'économie}

Confrontés à ce nouveau contexte, la Confédération et les cantons ont modifié leur politique de développement économique (réorientation de la politique régionale, de la promotion économique, appui au transfert technologique, nouvelle gestion publique, déréglemen- tation, etc.). De son côté, l'aménagement ne semble pas avoir pris en compte ces mutations économiques (certainement au niveau de l'analyse mais pas en terme de conséquence pour la pratique). 0 r, il doit s'interroger sur sa contribution à l'amélioration de la capacité concurrentielle de l'économie suisse et au développement durable du pays. A ujourd'hui, l'aménagement est perçu comme un coût et une succession de contraintes pour l'économie. II doit apporter la démonstration qu'il est source de gains et de sécurité, qu'il est facteur de «plus-value». Une telle exigence n'est possible que s'il est en mesure de prendre en compte, dans sa planification comme dans sa mise en oeuvre, ces exigences de rentabilité liées à tout investissement dans le secteur privé. Si tel ne devait pas être le cas, l'affectation du territoire prévue par l'acteur public ne coïncidera pas avec l'utilisation effective décidée par les acteurs privés. Le risque est alors que l'aménagement entre en conflit avec les exigences et les objectifs des politiques de développement économique; or il est vital que ces deux politiques demeurent complémentaires dans l'intérêt de l'économie suisse et de la capacité concurrentielle des entreprises et de la qualité de l'aménagement du territoire de notre pays.

Cette problématique interpelle clairement l'a ménagement dans le cadre des débats autour de l'avenir de la zone agricole: va-t-il se profiler uniquement en termes de prescriptions réglementaires à respecter dans le cas des bâtiments situés en zone agricole ou bien va-t-il prendre en compte l'avenir des entreprises et exploitations localisées dans cette zone et qui doivent se restructurer pour garantir leur capacité concurrentielle et donc leur survie?

Dans ce but, il s'agit d'examiner dans quelle mesure l'aména gement peut être conçu pour favoriser et même stimuler les capacités d'investissement dans les secteurs productifs et concurrentiels $d u$ pays. Autrement dit, pour intégrer la dimension de rentabilité, l'aménagement doit évaluer ses prestations et ses instruments pour voir si ceux-ci reconnaissent à l'investissement privé le rôle déterminant qu'il joue dans sa mise en $œ u v r e . C^{\prime}$ 'est à l'aménagement de s'adapter aux nouvelles réalités économiques et non l'inverse. Cette affirmation ne signifie pas une mise sous tutelle économique de l'aménagement qui doit veiller à ce que les autres préoccupations (écologiques, sociales, etc.), soient prises en compte. Elle concerne la manière dont est traitée la dimension économique.

Cette approche par l'investissement est sans doute nouvelle pour l'aménagiste. Elle ne l'est pas pour les responsables des politiques régionales et de promotion économique. Depuis les années 80 , ces dernières ont été conçues et mises en œuvre pour stimuler l'investissement à travers des appuis, conseils, mises en réseau et autres incitations. Ces politiques ont fait de l'acteur économique, et en particulier de l'entrepreneur-investisseur, un des acteurs-clés du développement régional. Il appartient à l'aménagement d'en faire un acteur stratégique - parmi d'autres - de la politique d'organisation du territoire. $\mathrm{M}$ ais est-ce dans la culture et dans la philosophie des aménagistes?

\section{La politique d'aménagement du territoire: à la recherche de nouvelles alliances}

D'abord un double rappel. Pendant les années de haute conjoncture, l'aménagement a bénéficié de la bonne santé des finances publiques, ce qui lui a permis de financer de nombreux projets et mesures favorables à sa mise en œuvre. Il suffit de penser aux politiques du logement et des transports. De plus, les collectivités ont réalisé de nombreux équipements collectifs qui ont servi à concrétiser des stratégies d'urbanisation. Or aujourd'hui, les finances publiques sont en crise: les investissements dans les infrastructures et équipements deviennent rares (il y a bien sûr quelques exceptions) et les problèmes se situent au niveau des coûts de fonctionnement. Même Rail-Bus 2000, un des piliers principaux de la politique d'aménagement pour notre pays, se joue à travers la négociation du financement des coûts de fonctionnement entre la Confédération, les cantons, les 
communes et les usagers. $0 \mathrm{r}$ dans cette négociation, l'aménagement peine à faire entendre son argumentation. Pourquoi donc l'aménagement ne s'intéresserait-il pas aux questions de finances publiques? II commence à le faire, car on constate que les coûts d'équipement et d'entretien sont en train de prendre l'ascenseur.

Deuxième rappel: l'aménagement, en Suisse, a été largement conçu et appliqué avec le souci de préserver les terres a gricoles (cf. les surfaces d'assolement); un des principaux objectifs a été de bien distinguer les zones agricoles et les terrains à bâtir. Cette préoccupation sera par la suite couplée avec celle de la protection de la nature, ce qui a conduit nombre de citoyens à penser que la politique d'aménagement avait comme objectif prioritaire la protection des zones agricoles et du paysage. Cette évolution sera encore renforcée par le fait que la protection de l'environnement va, dans les années 80 , bénéficier d'une forte légitimité sociale et politique au point de favoriser la mise en place d'une législation très normative. Protection de l'environnement et aménagement du territoire: les deux politiques ont fait alliance objectivement $M$ ais on doit aujourd'hui se demander si l'aménagement ne paie pas cette filiation par une perception ambiguë de son rôle et de ses instruments. II n'est pas rare qu'on les confonde, surtout dans leurs dimensions réglementaires. La pesée des intérêts est souvent assimilée aux résultats de l'étude d'impact. Là éga-lement, la perception ne correspond certainement pas à la réalité, mais elle contribue à la réputation réglementaire de l'aménagement auprès de la population.

Dans le contexte actuel, l'aménagement doit revendiquer sa fonction de coordination des politiques publiques (il l'exerce au niveau des projets concrets sur le terrain). Mais il fait partie des politiques «mineures», n'ayant plus la «cote politique», aussi est-il condamné, pour réaliser cette revendication, à faire alliance avec d'autres politiques publiques. II a un double choix (qui n'est pas exclusif):

- faire alliance avec des politiques secto rielles à fort impact spatial, comme les transports, la politique économique, la politique agricole; c'est le souci de la coordination fonctionnelle au niveau de la mise en œuvre opérationnelle de ces politiques publiques;

- s'associer à des politiques qui interviennent dans l'architecture institutionnelle de la Confédération, des cantons et des communes; c'est le souci de la coordination institutionnelle entre niveaux différents et de la prise en compte des compétences et des processus d'élaboration et de décision des politiques publiques.

$\mathrm{Ce}$ problème mérite une réflexion stratégique au sein des milieux de l'aménagement lorsqu'on constate, d'une part, que les services cantonaux d'aménagement sont attribués à des départements différents selon les cantons (il conviendrait de s'interroger sur les raisons et les conséquences de ces différences d'attribution), et d'autre part, que les propositions relatives à la future constitution fédérale, envisagent de confier la politique d'aménagement $d u$ territoire aux cantons.

\section{Et si l'aménagement raisonnait et travaillaiten termes de «prestations»?} Interrogez l'aménagiste travaillant dans un service public sur ses activités: il vous répondra qu'il planifie, coordonne, affecte, préavise, conseille. Complétez votre question sur la nature de ses prestations et leurs utilisateurs? La réponse devient plus floue et souvent elle interpelle l'interlocuteur. Les aménagistes se sentent généralement investis de la mission de défense de l'intérêt général en matière de gestion du territoire et leur activité est légitimée par la législation. Cette dernière définit les missions et conditionne l'organisation des services publics. N ombreux sont les services de I'administration qui, aujourd'hui, s'interrogent sur leurs prestations. Une base légale n'est plus suffisante pour justifier leur existence. Encore faut-il qu'ils fournissent des prestations qui correspondent à des besoins et à une demande des usagers. Q u'en est-il de l'aménagement?

Si certaines prestations se justifient par leur caractère obligatoire (par exemple, l'octroi d'une autorisation de construire), d'autres ne s'imposent que si elles constituent une plus-value pour celui qui y recourt (par exemple, le conseil pour concevoir et réaliser un projet de construction immobilière).

Rares sont les services d'aménagement qui ont identifié, de façon explicite, leurs prestations. De manière générale, elles peuvent être regroupées ainsi:

- les tâches de planification (aux différentes échelles institutionnelles);

- les tâches de coordination entre politiques sectorielles et/ ou au niveau des projets concrets d'a ména gement;

- les prestations réglementaires (octroi de permis de construire, acceptation/ préa vis concernant des plans);

- les tâches de conseil pour la mise en œuvre des plans ou le montage/ pilotage de projets à incidences spatiales;

- la mise en relation et en réseau d'acteurs concernés par un projet d'aménagement;

- les incitations financières et fiscales tant cantonales que fédérales (via I'AT ou via d'autres politiques);

- la production d'informations pertinentes pour l'organisation et la gestion du territoire.

Q ue peut-on constater au sujet de ces prestations?

- le service public d'aménagement est avant tout perçu comme un prestataire de décisions et d'actes juridiques et réglementaires. Pour le citoyen, il sanctionne des plans, il octroie des permis, délivre des autorisations de construire, décide des dérogations; cette perception est sans doute réductrice et fausse. Elle pénalise l'aménagement dans ses ambitions d'être le lieu où s'élabore et se débat un projet d'aménagement;

- les prestations de planification peinent à se profiler et à se faire reconnâ̂tre; les temps ne sont plus aux exercices de planification; au niveau cantonal, beaucoup d'espoirs sont mis dans le rôle du plan directeur cantonal en matière de coordination; mais quelles sont concrètement les prestations de coordination offertes par ce plan directeur? $\mathrm{N}$ 'est-il pas simplement un outil de coordination interne à l'administration cantonale et fédérale? Utilisation certes pertinente, mais qui ne peut être à la 
hauteur des ambitions évoquées précédemment pour l'aménagement;

- les informations: elles ne sont pas finalisées ni toujours actualisées et ont souvent un caractère général, ce qui les rend peu intéressantes, peu utilisables par les entreprises, les acteurs privés; quelle est leur «plus-value» pour une décision d'investissement?

- les prestations de conseil ont pris de l'importance mais elles sont mal connues et il est difficile d'être à la fois l'organisme de conseil et l'organisme de sanction;

- quant aux dispositions fiscales (prélèvement de la plus-value foncière), elles sont restées largement lettre morte dans la plupart des cantons.

La crise de légitimité de l'aménagement trouve largement son origine dans le fait que certaines de ses prestations sont remises en question ou même franchement rejetées alors que d'autres ne sont pas bien identifiées et reconnues.

Raisonner «prestations», c'est raisonner «destinataires» des prestations. C ette orientation permet d'identifier ces utilisateurs de manière plus précise et non seulement générale (il n'y a pas les entreprises, mais des PME, des multinationales, des entreprises artisanales, tertiaires, etc.). Elle permet de se demander si les prestations sont adaptées aux besoins de chaque type d'entreprises. Au moment où la société et l'économie sont en pleine mutation, cette demande est d'actualité. Enfin, en raisonnant «prestations» et «destinataires», il est alors possible d'examiner la pertinence et l'efficacité des instruments d'aménagement par rapport aux nouveaux comportements des acteurs publics et privés dans la société. Le logement permet d'illustrer le propos.

Dans les années de haute conjoncture, l'aménagement a planifié et réglementé l'affectation selon une certaine politique du logement (nécessité de logements sociaux, souci de maîtriser une demande pour le logement individuel, maîtrise des résidences secondaires). A ctuellement, les modes et les lieux d'habitat sont en pleine évolution avec l'émergence de nouveaux besoins (personnes âgées, famille monoparentale), l'écla tement des résidences secondaires non seulement en zones touristiques mais également en ville, le développement de la mobilité spatiale, etc. Le marché de la construction se restructure a vec l'apparition de nouveaux acteurs financiers (caisses de pension). La propriété privée gagne du terrain. Face à cette évolution, que dit, que propose l'aménagement du territoire, au-delà des questions de mixité et de desserte par les transports publics? Sait-il si ses instruments et sa pratique contribuent à réduire ou à accroître les inégalités sociales par rapport à l'accès au logement? Fournit-il, au-delà des inventaires statistiques, des informations pertinentes sur l'évolution du marché immobilier, sur les incidences de l'affectation sur la plus-value foncière, sur les acteurs gagnants et perdants de ce marché (tant du point de vue de l'offre que de la demande)?

En raisonnant «prestations», I'aménagement sera alors davantage en mesure d'intervenir en termes de priorités, de gains et de coûts. II cessera de développer des activités dont l'utilité n'est souvent pas évidente depuis l'extérieur pour promouvoir des prestations répondant aux besoins des acteurs du territoire. Il contribuera à clarifier les enjeux spatiaux et les inévitables conflits pour l'appropriation du sol en fournissant des informations pertinentes aux acteurs du jeu de l'aménagement.

\section{Notes}

[1] Ces réflexions s'appuient sur une bibliographie, dont nous avons donné les principaux titres en fin d'article.

\section{Bibliographie}

Decoutère, S., J. Ruegg, et D. Joye (éds), 1996, Le management territorial. Pour une prise en compte des territoires dans la nouvelle gestion publique. Lausanne: PPUR.

Fingerhuth, C., 1998. Städtebau ist Raumplanung für die Stadt. Bern. Bulletin d'information de l'O ffice fédéral de l'aménagement du territoire 1/ 98:17-19.
Keller, D.A., M. Koch, K. Selle (Hsg), 1998, Planung + Projekte. Verständigungsversuche zum W andel der Planung. Dortmund. Dortmunder Vertrieb für Bau- und Planungsliteratur.

Lendi, M.,1996. Grundriss einer Theorie der Raumplanung. Einleitung in die raumplanerische Problematik. Hochschulverlag AG ETH Zürich.

Meier, R., 1996, Die Raumplanung im Lichte der Privatisierung, Liberalisierung, Deregulierung und Dezentralisierung. Disp 96: $20-25$.

Rey, M. 1994. La gestion du processus de décision en aménagement du territoire. Lausanne: C.E.A.T. Polycopié.

Rey, M., 1997. Gestion des processus de changement en matière de développement régional. Disp 131: 14-21.

Ruegg, J., 1997, O rigine, portée et enjeux du zonage: propriétaires en zone à bâtir. Faculté des SSE, Université de Lausanne, thèse.

Ruegg, J., S. Decoutère et N. M ettan (éds), 1994, Le partenariat public-privé: un atout pour l'aménagement du territoire et la protection de l'environnement? Lausanne: PPUR Ruegg, J., 1991, M édiation des conflits d'utilisation du sol et a ména gement postmoderne. Disp 106: 28-33.

Ruegg, J., 1988, Les intérêts particuliers: I'essence de l'aménagement du territoire. Disp 94: 5-9.

W alter, F., 1997, Cinquante ans d'a ménagement $d u$ territoire en Suisse: quelques questions aux acteurs. Disp 127: 35-40. 


\section{Stellungnahmen zu den Thesen} zur Raumplanung von Michel Rey

Bernard Staub, dipl. Geograph, Raumplaner ETH/ NDS, Chef das Amtes für Raumplanung Kanton Solothurn

Regelmässig werden Versuche unternommen, die Rolle der Raumplanung zu analysieren, zu kommentieren und Schlüsse für die künftige Ausrichtung zu ziehen. Dieses Unterfangen zeitigt - je nach Herkunft des Autors - unterschiedliche Resultate. So hat vor kurzem Professor M artin Lendi - ein profunder Kenner der Raumplanungsszene - bei der Vorstellung des neuen Vademecums Raumplanung Schweiz durch das Bundesamt für Raumplanung von der Erfolgsgeschichte Raumplanung gesprochen, ohne jedoch die Schwierigkeiten raumplanerischen Bemühens zu negieren. $\mathrm{N}$ un konfrontiert uns $\mathrm{M}$ ichel Rey mit Thesen zur aktuellen Situation der Raumplanung. Er spricht von der Krise der Raumplanung, er identifiziert einen Verlust an G laubw ürdigkeit, er kritisiert die fehlende Anpassungsfähigkeit der Raumplaner an veränderte Rahmenbedingungen und ortet schliesslich sogar ein Feinbild $z$ wischen $W$ irtschaft und Raumplanung.

Die Grundsatzrage «Raumplanung wohin?» stellt sich. Mit dieser Herausforderung müssen wir Raumplaner leben. W ir wissen auch, das einzig stetige ist die Veränderung. Bewähren muss sich die Raumplanung bei der Lösung konkreter Probleme. A ufgrund der laufenden raumplanerischen Aktivitäten in einem mittelgrossen Kanton sehe ich in der Rolle und im Stellenwert, den die Raumplanung einnimmt, durchaus positive Ansäte für mehr Selbstbewusstsein in dieser Disziplin.

Aber vorweg, weshalb kommen derart unterschiedliche Beurteilungen zustande? Ist es einzig eine Frage des Blickwinkels? Eine bis zur Mitte mit Flüssigkeit gefüllte Flasche ist je nach Standpunkt halbleer oder halbvoll. Beide Sichtweisen sind im $\mathrm{G}$ runde genommen «richtig», allerdings haben die Betrachter einen unterschiedlichen Blickwinkel.

\section{Akzeptierte staatliche Kerna ufgabe}

Heute stellen wir fest, dass die raumplanerische Euphorie der Anfangsjahre einer nüchternen und realistischen Einschä Łung der Möglichkeiten gewichen ist. Die Raumplanungsaufgaben wahrzunehmen und deren Leistungen zu kommunizieren ist nicht immer einfach. Raumplanung nimmt in der politischen Einschätzung und Diskussion keinen Spitzenplatz mehr ein. Das konnten wir kürzlich bei der Revision des Raumplanungsgesetzes feststellen: Die Debatte im Parlament verlief relativ lustlos. Der niedere politische Aktualitätswert der Raumplanung wird von einigen Exponenten bedauert. Das ist die eine Seite der Medaille.

Aber auf der anderen Seite können wir mit Befriedigung feststellen, dass die Raumplanung als Kernaufgabe des Staates ihren Platz eingenommen hat. Sie ist rechtlich und instrumentell abgestützt und akzeptiert. Auch in einem sich ändernden gesellschaftlich-politischen Umfeld haben die G rundkonstanten der Raumplanung nichts von ihrer ursprünglichen A ktualität verloren: Die «Trennung von Siedlungs- und $\mathrm{N}$ ichtsiedlungsgebiet» und das «Schaffen gleichwertiger Lebensbedingungen» sind Ziele, die in der Zwischenzeit durch neue, wie beispielsweise die zweckmässige Vernetzung unserer Städte, die Steigerung der Siedlungsqualität und die nachhaltige $\mathrm{N} u$ tzung des ländlichen Raumes ergänzt worden sind.

\section{Prozessorientierte Raumplanung}

Im Kanton Solothurn sind wir bestrebt, die Anregungen aus der laufenden Raumplanungsdiskussion a ufzunehmen und in das dynamische, räumliche Koordinations- und Führungsinstrument «Kantonaler Richtplan» einzubauen. W ir haben uns bewusst auf die prinzipiellen $\mathrm{N}$ utzungsz uw eisung en konzentriert, klare Leitplanken definiert und dadurch viel Ballast abgeworfen. Mit der Konzentration auf das Wesentliche haben wir Schwerpunkte gesetzt, um einen möglichst effizienten Einsatz der beschränkten öffentlichen Mittel (z.B. im Bereich öffentlicher Verkehr) im Hinblick auf die angestrebte Raumordnung zu erreichen. $M$ it der Bezeichnung von überörtlichen Arbeitsplatzgebieten konnten klare Signale ausgesendet werden. Die W irtschaft weiss, dass aus kantonaler Sicht an diesen Standorten die Unterstützung der A mtsstellen sichergestellt ist.

Das Rezept für eine erfolgreiche verwaltungsinterne Abwicklung komplexer Bewilligungsverfahren kann unter den Stichworten «klare Führung» und «umfassende Koordination» zusammengefasst werden. Im Kanton Solothurn lösen wir diese Aufgabe wie folgt: Für die Verfahrensleitung wird ein Projektleiter G esamtkoordination aus dem Amt für Raumplanung und ein Teilprojektleiter Umwelt aus dem Amt für Umweltschut bestimmt. Beide verfügen über weitgehende Kompetenzen. Die speditive Abwicklung wird durch eine interdepartementale Chefbeamtenkonferenz beaufsichtigt.

Diese koordinierte Verfahrensleitung ist eine Dienstleistung des Kantons; sie hat sich bereits bei verschiedenen Projekten (z.B. Postpaketzentrum Härkingen) sehr gut bewährt. A ber auch ohne konkretes Bauvorhaben wird in ausgewählten überörtlichen Arbeitsplatzgebieten die Planung mit den Hauptbeteiligten ( $G$ rundeigentümer, Stadt, Kanton) vorang etrieben, damit auf entsprechende Anfragen rasch reagiert werden kann. Raumplanung ist Teil einer positiven (wirtschaftlichen) Entwicklungsplanung $z$ wischen privaten und öffentlichen Stellen.

\section{Verstärkung der Kommunikation}

Sinn und Zweck der Raumplanung müssen ständig erklärt werden; sie erklären sich nicht von selbst. W ir müssen mit viel Engagement und mehr Aufwand aufzeigen, dass es sich nicht um eine abschliessend definierte Staatsaufgabe handelt. Die Erarbeitung des kantonalen Richtplanes, die Überarbeitung der $O$ rtsplanungen oder die $M$ itarbeit an der Planung und Umsetzung eines konkreten Bauvorhabens sind günstige Gelegenheiten, um unsere Aufgabe bekannt zu machen. Eine offene, rechtzeitige und kompetente Kommunikation unterstützt unsere Arbeit. Die Zusammenarbeit mit den verschiedensten Interessengruppen ist ein Muss. Der Abbau von Vorurteilen tut not und der Aufbau persönlicher Kontakte führt in vielen Fällen zu erfolgversprechenden Ansätzen. Das alles braucht viel Zeit. Diese ist 
aber vielfach nicht vorhanden. Deshalb ist der Kommunikationsprozess ein nicht zu unterschätendes permanentes Standbein unserer Tätigkeit: $W$ ir müssen unsere Anliegen in Erinnerung rufen, und gleichzeitig erfahren wir frühzeitig von Ideen und (Bau-)Absichten. Das setzt voraus, dass wir die wesentlichen raumplanerischen Leitplanken - sei es verfahrensmässig oder inhaltlich definiert haben. Die Partner wollen eindeutige Aussagen, sie wollen wissen, was machbar ist. Die Raumplanung muss für bestimmte $\mathrm{G}$ ebiete $\mathrm{M}$ öglichkeiten aufzeigen, sie muss aber gleichzeitig an anderen 0 rten auch klare $G$ renzen setzen - dies alles mit dem nötigen Selbstbew usstsein.

Dr. Fritz. W egelin,

Bundesamt für Raumplanung

\section{Weder Klagelieder noch Selbst- zufriedenheit sind am Platz}

\subsection{Richtig gezielt und getroffen, aber nicht ins Schwarze}

Die Debatte ist nötig. Der Beitrag von Michel Rey eignet sich durchaus, die Raumplanung etwas gegen den Strich zu bürsten. M. E. weist er zu Recht auf Entwicklungen hin wie der nachlassende Elan der Raumplanung, die G efahr inrer Verrechtlichung oder das Defizit an Koordination mit den Sektoralpolitiken. Auch wenn er fordert, die Raumplanung solle ihre Instrumente überprüfen, das Verhältnis zur «W irtschaft» und zur wirtschaftlichen Entwicklung klären, verstärkt prozessorientiert und kundenorientiert handeln, sich mit den neuen Herausforderungen auseinandersetzen und strategische Allianzen eingehen, stimme ich ihm zu.

Andere Aussagen halte ich jedoch für unzutreffend, so z.B. die Raumplanung kümmere sich nur um Landwirtschaft und Landschaft und nehme die Bedürfnisse der W irtschaft nicht wahr, sie nehme die neuen Entwicklungen nicht zur Kenntnis und beharre auf ihren traditionellen, überholten Instrumenten, sie in- teressiere sich nicht für öffentliche Finanzen, missbrauche die Mitwirkung der Bevölkerung bei der Planung, um Projekte besser «verkaufen» zu können, und leide unter einem Defizit an politischer und sozialer Legitimation.

Insgesamt halte ich das in der Darstellung vermittelte Bild als zu einseitig, zu pessimistisch und nicht ganz auf dem aktuellen Stand. In den Ausführungen fehlen mir insbesondere die Auseinandersetzung mit den Zielen und mit grossen Themen wie nachhaltige Entwicklung, Rolle und Probleme der Städte und Agglomerationen, Mobilitätsfragen (offenbar ein Tabuthema), Einsatz marktwirtschaftlicher Instrumente, bessere Koordination im Lebensraumrecht mit Blick auf eine echte Straffung der Verfahren sowie die europäische Raumordnung [1].

\subsection{Raumplanung hat Ziele}

Der Verfasser der «Thesen» sieht die Raumplanung vorwiegend instrumentell. Es gibt aber nicht nur Spielregeln, Verhandlungen, Moderation. Raumplanung wurde in die Verfassung hineingeschrieben, weil man ganz bestimmte Probleme lösen und Ziele erreichen wollte. Raumplanung ist nicht einfach nur Interessenausgleich von Fall zu Fall, sondern zielgerichteter Interessenausgleich. Die Debatten um die Grundzüge der Raumordnung und die Revision des Raumplanungsgesetzes haben gezeigt, dass die grundlegenden Ziele nach wie vor breite Akzeptanz finden. Die zweckmässige Nutung des knappen und unvermehrbaren Bodens, eine geordnete Besiedlung, eine funktionierende Raumstruktur sowie faire und effiziente Verfahren für einen zielgerichteten Interessenausgleich sind nach wie vor ökologisch, gesellschaftlich, wirtschaftlich und ethisch fundiert.

\subsection{Raumplanung ist besser als ihr Ruf, aber sie muss Leistungsa usweise erbringen}

Raumplanung hat zweifellos Erfolge zu verzeichnen. Das gibt auch Rey vor seinem grossen ABER zu. Ich glaube, wir haben zu viel über Misserfolge gejammert und dabei übersehen, dass Raumplanung gesamthaft betrachtet eine
Erfolgsstory ist. Wenn man das W achstum der Ansprüche an den Lebensraum der letzten 20 - 30 Jahre vor Augen hat, so dürfen sich ihre Leistungen durchaus sehen lassen. In dieser Zeit hat sie sich zudem auch als öffentliche A ufgabe etabliert, und öffentlich-rechtliche Eigentumsbeschränkungen sind selbstverständlich gew orden [2].

Die Tatsache, dass Raumplanung nicht mehr zuoberst auf der politischen Traktandenliste steht, ist nicht a priori als ein schlechtes Zeichen zu werten. Zuoberst stehen nämlich immer jene Politikbereiche, welche die grössten Probleme aufweisen. In den 50 er und 60 er Jahren war es die Raumordnung, später der Umweltschut, die Migrationsfragen, die Staatsfinanzen, die Sozialpolitik, die Arbeitslosigkeit. Der Rückzug aus den Schlagzeilen darf nicht mit mangelndem Interesse an längerfristigen Prioritäten verwechselt werden [3]. $M$ an muss a uch unterscheiden $z$ wischen dem, was eine M ehrheit der Bürger will und dem Lärm, der von einer kleinen interessengeleiteten $M$ inderheit ausgeht, weil sie mit den demokratisch abgestüt ten Plänen nicht einverstanden ist.

Trotzdem - hier gebe ich Rey recht zu Selbstz ufried enheit ist kein A nlass. Es ist den Raumplanern zu wenig gelungen, ihren Beitrag an eine gedeihliche und nachhaltige Entwicklung genügend sichtbar zu machen; ein Leistungsausweis ist ohne Einschaltquotendenken ständig zu erbringen und $z u$ kommunizieren, wenn man politisch legitimiert bleiben will.

\section{Neubelebung und Neuausrichtung}

Eine $\mathrm{N}$ eubelebung und $\mathrm{N}$ euausrichtung halte ich - wie Rey - angesichts neuer und veränderter Verhältnisse für notwendig. Nur setze ich die Akzente etwas anders.

\subsection{Nachhaltige Raumentwicklung}

Da sich Rey nicht mit den Zielen der Raumplanung auseinandersetzt, hat er auch die aktuelle Diskussion um die $\mathrm{N}$ achhaltigkeit ausgelassen. Das deutsche Raumordnungsgeset hat die nach- 
haltige Raumentwicklung zur Leitvorstellung erhoben. Die Grundzüge der Raumordnung Schweiz [4] wie auch das Europäische Raumentwicklungskonzept[5] der Europäischen Union haben sie ins Zentrum gesetzt. Eigentlich war die Raumplanung implizit immer schon den Prinzipien der nachhaltigen Entwicklung verpflichtet: 0 ptionen freihalten für nachfolgende Generationen sowie «die sozialen und wirtschaftlichen Ansprüche an den Raum mit seinen ökologischen Funktionen in Einklang bringen und zu einer dauerhaften, grossräumig ausgewogenen Raumordnung führen»[6]. Dennoch glaube ich, dass die Diskussion um die $\mathrm{N}$ achhaltigkeit für die Raumplanungsziele neue Akzente zu setzen vermag. Klassische Raumplanungsziele wie gleichwertige Leb ensverhältnisse und dezentralisierte Konzentration gelten zwar immer noch, aber sind unter dem Aspekt der $\mathrm{N}$ achhaltigkeit in einem anderen Licht $z u$ betrachten [7]. Unter diesem Aspekt erweist sich die von Rey postulierte Ausrichtung auf die Interessen der $G$ rundeigentümer und Investoren als eine $z u$ enge und $z u$ kurzfristige 0 ptik. Offenheit für die Zukunft verstärkt eher die ökologische Komponente. Das Thema kann nicht der Umweltpolitik allein überlassen werden. Eine prozessorientierte Raumplanung vermag einen bedeutenden Beitrag zur Umsetzung der $\mathrm{N}$ achhaltigkeits-Ziele $\mathrm{zu}$ leisten [8].

\subsection{Raumplanung und W irtschaft umfassend betrachten}

Zur nachhaltigen Entwicklung gehört auch die ökonomische Dimension. Raumplanung hat unter anderem auch den A uftrag, «die räumlichen Voraussetzungen für die W irtschaft zu schaffen». Ich stimme Rey zu, dass hier Defizite und Berührungsängste bestehen. Ein besserer Dialog zwischen Raumplanung und $W$ irtschaft tut not. Bund und Kantone bleiben jedoch nicht untätig. Die Beispiele für eine gute Zusammenarbeit mehren sich und die Verfahren wurden und werden noch verbessert. Eine gute Standortvorbereitung - unter W ahrung des langfristigen Grundauftrages für eine zweckmässige N utung des Bodens, geordnete Besiedlung des Lan- des zu sorgen - nützt auch der W irtschaft. Dazu bedarf es einer über einzelbetriebliche $W$ ünsche hinausgehende, längerfristige Sichtweise, ganz im Sinne einer nachhaltigen Raumentwicklung, die ja neben der wirtschaftlichen auch eine soziale und ökologische Dimension enthält. In diesem Zusammenhang spielen die Städte und Agglomerationen, die grossräumigen Erschliessungen, die europäischen Verbindungen und die Infrastrukturnetze eine bedeutende Rolle.

Reys Überlegungen sind eher betriebswirtschaftlich als volkswirtschaftlich begründet. Dabei lässt sich gerade hier zeigen, dass Markt und Raumplanung keine $G$ egensätze zu sein brauchen. Marktwirtschaftliche $M$ assnahmen können zwar Raumplanung nicht ersetzen, aber sie unterstützen grossmehrheitlich ihre Ziele [9]. Allerdings können auch spannende Fragen auftauchen. A ktuell ist beispielsweise die Diskussion, inwieweit Liberalisierungstendenzen in zahlreichen raumwirksamen Politikbereichen wie öffentlicher Verkehr, Energie, Kommunikation in dünn besiedelten ländlichen Räumen und im Berggebiet den Service public gefährden und ob Massnahmen zur Sicherstellung einer genügenden $G$ rund versorgung ins Auge gefasst werden müssen. Eine Möglichkeit besteht $\mathrm{z}$. B. in der $\mathrm{N}$ euorientierung des bundesstaatlichen Finanzausgleichs, soll doch dieses Projekt dafür sorgen, die schwächeren $\mathrm{G}$ ebiete in die Lage zu versetzen, durch eine genügende Ausstattung an finanziellen Mitteln einen $M$ indeststandard an Leistungen erbringen zu können.

\subsection{Internationale A usrichtung}

Heute ist die Einbindung der Schweiz in die europäische Raumordnung zu einer von vier Hauptstossrichtungen der schweizerischen Raumordnungspolitik geworden. N eben der Schaffung einer zweckmässigen Raumstruktur für eine nachhaltige internationale W ettbewerbsfähigkeit geht es sowohl um die Einbindung der Schweiz in die europäischen Städte- und Infrastrukturnetze (Verkehr, Kommunikation, Versorgung), aber auch um die Zusammenarbeit unter ländlichen und alpinen $G$ ebieten Europas und den Beitrag der Schweiz an eine europäische Raumordnung.

\subsection{N eua usrichtung der Instrumente und neue Formen der Planung}

Der Vorwurf, die Raumplanung arbeite stur mit überholten Instrumenten und kümmere sich nicht um die öffentlichen Finanzen, trifft immer weniger zu. Die Bund esraumplanung ist z. B. überwiegend ausserhalb der traditionellen Instrumente tätig und kümmert sich ebenfalls um finanzielle Fragen. Die bisherigen Instrumente der Raumplanung sind notwendig, aber nicht hinreichend zur Erreichung der Ziele. W ill Raumplanung von ihrer nachvollziehend defensiven, nominalen Haltung wegkommen und sich in Richtung einer funktionalen raumordnungspolitischen Aufgabe bewegen, die stärker prospektiv und offensiv operiert, bedarf es ebenso der $\mathrm{N}$ euausrichtung und veränderten Ausgestaltung bestehender Instrumente wie auch neuer Formen. Die O ffenheit gegenüber der Zukunft bedingt eine prozessorientierte Planung. Dabei müssen die raumordnungspolitische und raumplanerische Koordination, die A ushandlungsprozesse, die Terra invorbereitung für N utzungen und Umnutzungen, die Implementation sowie das Controlling verbessert werden.

\subsection{Intellektuell und kommunikativ verknüpfen}

Jede Disziplin macht Veränderungen mit. In der Raumplanung ergeben sich Verschiebungen von einer eher statischen Sicht der Bodennutzungsplanung zu einer eher strategischen, prozessorientierten Ausrichtung. Jener Planer wird die $\mathrm{N}$ ase vorn haben, der nicht irgendeinem M odetrend nachrennt oder sich dem Jammern hingibt, sondern der über die notwendige intellektuelle und kommunikative Vernetzungsfähigkeit verfügt. Er muss die räumlichen Zusammenhänge aufzeigen und einzelne raumwirksame Anliegen an der Leitidee für eine gesamträumliche nachhaltige Entwicklung orientieren und dazu die Zusammenarbeit fördern. Seine Hauptaufgabe besteht darin, die verschiede- 
nen Interessen zusammenzuführen und in einen Gesamtrahmen zu stellen. $\mathrm{Er}$ muss die richtigen Leute an den Tisch holen. W enn ich Rey richtig verstanden habe - und es war nicht ganz einfach -, sollten wir uns in diesem Punkt einig sein.

\section{Anmerkungen}

[1] Eine umfassende, zukunftsorientierte Darstellung zum Wandlungsprozess der Raumplanung und zu deren Herausforderungen gibt Lendi, M., Raumplanung im Umbruch A uf dem W eg zu einer politisch, sachlich und ethisch indizierten Raumplanung. Erweiterte Fassung des Vortrages, gehalten am 18. Mai 1998, vor der Europäischen Bodenfakultät an der ETH Zürich; vgl. auch Lendi Martin, Politisch, sachlich und ethisch indizierte Raumplanung, am Beispiel der Schweiz, W ien 1998

[2] vgl. ebenda

[3] vgl. Vera Herrmann/Gian-Reto Raselli, Forschungsinstitut der G esellschaft für Sozialforschung (GFS), Regionale Identifikation und Stellenwert der Raumplanung aus Bürgersicht, in: Raumplanung, Informationsheft 3/97 des Bundesamtes für Raumplanung, S. $9 \mathrm{ff}$.

[4] Schweizerischer Bundesrat: Bericht über die Grundzüge der Raumordnung Schweiz vom 22. Mai 1996, Bern

[5] EUREK/ Europäisches Raumentwicklungskonzept. Erster offizieller Entwurf, vorgelegt bei dem informellen Treffen der für die Raumordnung zuständigen Minister der M itgliedstaaten der Europäischen Union, N oordwjik 1997

[6] Bundesraumordnungsgesetz $1998 \mathrm{der}$ Bundesrepublik Deutschland vom 18.8.1997, $\S 1$ Abs. 2

[7] Thierstein A., Lamprecht M., Raumordnung und nachhaltige Entwicklung: Handlungsansätze für eine nachhaltige Raumentwicklung in der Schweiz, Hrsg: EJPD/EVD, Bundesamt für Raumplanung/Bundesamt für W irtschaft und Arbeit, Bern 1998

[8] vgl. Lendi, M., ebenda

[9] E. Staehelin/ Hj. Bloechliger/ R. L. Frey, $M$ arktwirtschaftliche Reformvorschläge: Unterstüten sie die Raumplanung?, Hrsg. Bundesamt für Raumplanung, Bern 1995
Pierre-A lain Rumley, aménagiste, professeur à I'EPFL

Il faut savoir gré à Michel Rey de vouloir susciter un débat sur l'a ménagement du territoire, et ce parmi les aménagistes eux-mêmes. Cela étant, je ne partage pas un certain nombre de ses affirmations, tels que l'heure est «au doute», que «l'aménagement est en perte de vitesse», que «les aménagistes s'interrogent sur leur rôle et sur leur pratique», que «le territoire est le grand oublié de la restructuration et avec lui l'aménagement», que «la mise en œuvre des plans communaux adoptés se heurte à des difficultés: les affectations planifiées par l'autorité ne correspondent plus aux utilisations souhaitées par les propriétaires!», ou encore que ces "affirmations (... ) résument bien le débat d'idées a utour de la crise actuelle de l'aménagement du territo ire en Suisse».

La plupart de ces assertions ne me paraissent pas démontrées et je me risquerais même à formuler les thèses inverses suivantes, que m'inspirent une assez longue pratique de l'aménagement du territoire en Suisse et l'appréciation de la situation en Europe:

- L'importance de l'aménagement du territoire n'a cessé de croître depuis le début des années 80 .

- Les instruments de l'aménagement du territoire sont maintenant établis et reconnus. II en est de même de ses buts. - Les aménagistes participent largement au débat d'idées sur l'avenir de la Suisse (participation active à la collaboration transfrontalière, promotion des villes, devenir des agglomérations, avenir de l'espace rural, etc.

- Les aména gistes se sont montrés innovatifs et innovateurs dans l'adaptation de leurs méthodes et techniques (instruments en particulier).

II n'y a donc aucune raison d'évoquer une crise de l'aménagement du territoire.

Il est normal et souhaitable d'adapter ses pratiques tant et aussi longtemps que l'on ne perd pas de vue la raison d'être de son activité. II est dans l'ordre des choses que tous les propriétaires ne puissent pas utiliser leurs parcelles comme ils le souhaiteraient.
L'aménagement du territoire poursuivant des objectifs fort divers (cf. par ex. les buts et principes selon la LAT) impliquant en permanence des arbitrages politiques et des pesées d'intérêts, il serait fort étonnant qu'il ne prête pas flanc à la critique.

Il importe également d'être à la fois ambitieux et modeste dans l'appréciation du rôle que peut jouer l'aménagement $d u$ territoire qui n'est finalement qu'une politique publique parmi d'autres.

Je ne suis pas certain non plus que l'aménagement devrait tirer toutes les conséquences $d u$ fait que les temps ont changé, soit sans doute que nous devrions nous adapter à la globalisation.

$Q$ uelles conséquences devraient être tirées? N e s'agit-il pas plutôt d'anticiper sur les problèmes de demain, en participant pleinement au débat sur le développement durable, où il s'agit de concilier les impératifs économiques, sociaux et environnementaux? Le développement durable ne constitue-t-il pas une stratégie très proche des préoccupations de l'aména gement du territo ire?

Il est vrai que ce dernier necessite en permanence une prise en compte de nouveaux besoins et de nouvelles idées, ce qui implique une adaptation périodique des plans. II convient toutefois de ne céder ni aux pressions injustifiées ni aux modes.

Je reste convaincu quant à moi que la société a besoin d'aménagistes qualifiés (et nous avons dans ce domaine des progrès à faire en Suisse) et engagés, c'est-à-dire défendant un certain nombre de valeurs fondamentales. Je ne crois pas à l'aménagiste «fonctionnaire et neutre», se contentant d'arbitrer entre les intérêts en présence. Je ne connais pas de bon amènagiste qui ne soit pas motivé par la protection de l'environnement, de la nature et du paysage, ce qui ne signifie pas qu'il faille limiter ses a miances à cette seule problématique.

Je partage largement l'avis de $\mathrm{M}$ ichel Rey au sujet de la nécessité de préciser les prestations de l'a ména gement du territoire. C'est d'ailleurs une tâche à la quelle les services d'a ménagement du territoire et d'urbanisme sont confrontés à l'occasion des réformes de l'administration et du N PM. 
D'autres tâches me paraissent prioritaires:

- la recherche

- a formation (de base et continue)

- la réflexion sur le développement durable

Deux défauts des aménagistes me paraissent devoir être combattus:

- Le premier est celui de vouloir faire de l'aménagement du territoire, vu sa problématique globale, une tâche publique prioritaire (ou «la» tâche publique par excellence).

- Le second est cette propension à suivre des modes, à évoquer des crises qui n'existent pas (ou en tout cas pas plus que dans d'autres disciplines), à changer trop rapidement certaines pratiques ou la conception de certains instruments, à mettre plus l'accent sur les divergences que sur les convergences dans la pratique professionnelle.

$\mathrm{N}$ ous devrions au contraire vouer to us nos efforts au développement d'une culture de l'aménagement du territoire, au travers d'un travail de fond dans les écoles, les universités, d'une meilleure information, d'une plus grande solidarité professionnelle et d'un meilleur marketing. 